\title{
The superparamagnetism of Yucca Mountain Tuff
}

\author{
Horst-Ulrich Worm \\ Federal Institute for Geosciences and Natural Resources, Hannover, Germany \\ Mike Jackson \\ Institute for Rock Magnetism, School of Earth Sciences, University of Minnesota, Minneapolis, \\ Minnesota
}

\begin{abstract}
Yucca Mountain Tuff contains small titanomagnetite grains with narrow size distributions in the superparamagnetic range [Schlinger et al., 1988]. Magnetic measurements on three samples (comprising hysteresis loops at low and ambient temperatures, acquisition and demagnetization of isothermal remanent magnetization, thermal demagnetization of the saturation remanence and of a low-temperature thermoremanence, and frequency- and temperature-dependent susceptibilities) allow evaluation of the magnetic properties in terms of Néel's [1949] single-domain theory. Precise grain volume distributions have been obtained by applying the blocking volume concept to thermal demagnetization results. In contrast, an attempt to derive mean particle volumes by fitting a Langevin curve to the room temperature magnetization curves fails, probably because the precondition for the Langevin function, $K V / k T \ll 1$, is not met. It is only for the sample with the smallest grains and in weak fields $(<20 \mathrm{mT})$ that a Langevin fit provides a reasonable volume estimate. There is good agreement between the experimental results and the calculated frequency and temperature dependence of susceptibility, thus verifying that Néel's theory is sufficient for the magnetic description of single-domain assemblages spanning the superparamagnetic/stable single-domain boundary. However, some deviations between modeled and measured susceptibilities exist, and the physical causes may include size-dependent anisotropy, nonuniform magnetizations, and also an uncertain preexponential time "constant" $\tau_{0}$. While $\tau_{0}=10^{-11} \mathrm{~s}$ gives the best fit for the sample with the largest grains, $\tau_{0}=10^{-9} \mathrm{~s}$ is more reasonable for the others. Thus $\tau_{0}$ may indeed be size- and temperature-dependent as predicted by Brown [1959]. The commonly cited parameter $\chi_{\mathrm{fd}}$ (frequency dependence of susceptibility) reaches $30 \%$ at room temperature (RT) for one sample with a blocking temperatures just below RT, while $\chi_{\mathrm{fd}}=0$ at RT for a superparamagnetic sample with smaller grains. These results thus exemplify that $\chi_{\mathrm{fd}}$ is not limited to $15 \%$, as a number of studies suggest, and that $\chi_{\mathrm{fd}}=0$ must not be taken to imply the absence of superparamagnetic grains.
\end{abstract}

\section{Introduction}

The properties of small magnetic grains change dramatically at the superparamagnetic (SP) to stable singledomain (SSD) threshold. SSD grains are extremely stable carriers of magnetic remanence because magnetization reversals require fields that are orders of magnitude larger than the geomagnetic field. Below a critical grain volume (or above a critical temperature), the SSD grains become superparamagnetic as thermal agitation rapidly (with respect to the timescale of interest) overcomes magnetic anisotropy,

Copyright 1999 by the American Geophysical Union.

Paper number 1999JB900285.

0148-0227/99/1999JB900285\$09.00 and these grains lose their net remanence. Conversely, the ability to equilibrate rapidly with a changing applied field endows SP particles with very high magnetic susceptibilities. SSD susceptibility arises from small rotation of grain magnetic moments toward an applied field direction, the rotation being strongly limited by particle anisotropy. In contrast, SP susceptibility involves complete rotation of particle moments into alignment with an applied field, the alignment being perturbed only by thermal fluctuations.

The SP/SSD threshold size depends on the mineral's spontaneous magnetization, its anisotropy, and the characteristic time of measurement, which may range from $<10^{-7} \mathrm{~s}$ to $>1000 \mathrm{~s}$ [e.g., Dormann, 1981]. As a consequence of this dependence, susceptibility may vary significantly with the frequency of an applied ac field in samples with a significant population of SP particles. The 
frequency dependence is typically quantified by the parameter $\chi_{\mathrm{fd}}[\%]=\left[\left(\chi_{\text {lf }}-\chi_{\mathrm{hf}}\right) / \chi_{\text {lff }}\right] \times 100$, where $\chi_{\text {lf }}$ and $\chi_{\mathrm{hf}}$ are the respective susceptibilities measured at low and high frequency $(470 \mathrm{~Hz}$ and $4.7 \mathrm{kHz}$ for the widely used Bartington ac bridge).

The theory of superparamagnetism dates back to Néel [1949], and its validity is not questioned. However, there is a commonly observed feature in the superparamagnetism of rocks and sediments that is not predicted by Néel's theory, namely, an apparent ceiling for the frequency dependence of susceptibility. Measurements on a large number of samples from various sources appear to indicate an upper limit for $\chi_{\mathrm{fd}}$ 15\% [Thompson and Oldfield, 1986; Dabas et al., 1992; Forster et al., 1994; Dearing et al., 1996]. This apparent limit is related to the distribution of particle sizes near the SP-SSD boundary, as shown by the calculations of Stephenson [1971], using a power law volume distribution $N(V) \propto V^{-2}$. This sort of distribution is clearly not realistic (since $N \rightarrow \infty$ as $V \rightarrow 0$ ), but it is a useful approximation for the upper tail of a normal or lognormal distribution in the special case where most grains are below the SP/SSD threshold. More generally applicable calculations of $\chi_{\mathrm{fd}}$ performed by Eyre [1997], using lognormal volume distributions, showed that sufficiently broad distributions limit $\chi_{\mathrm{fd}}$ to $15 \%$ or less regardless of the modal volume but that $\chi_{\text {fd }}$ may reach $90 \%$ for narrow distributions centered near the SP-SSD critical size. The calculated $\chi_{\mathrm{fd}}$ values in that study were erroneously large for a variety of reasons, and as a result, the broadness of the size distributions required to limit $\chi_{\mathrm{fd}}$ to $15 \%$ was also excessive. Nevertheless, the calculations of Worm [1998], incorporating a distribution of coercivities, lognormal volume distribution, and a gradual transition from SP to SSD susceptibility, still showed that $\chi_{\mathrm{fd}}$ can be larger than $15 \%$ for relatively narrow size distributions and that reasonably broad distributions can account for the observed limit.

Model calculations have thus shown that it is possible to reconcile limited $\chi_{\text {fd }}$ with Néel's theory in a somewhat ad hoc way, by calling on broad size distributions. However, there remains a lack of critical experimental data to rigorously test the underlying theory by establishing the frequency dependence of samples with independently characterized nartow size distributions. This lack is primarily due to the great difficulty of obtaining suitable natural or synthetic samples. The sizes of ferrimagnetic grains in rocks most often cover several orders of magnitude in diameter, and rocks are therefore generally not suitable for the study of grain-size-dependent magnetic parameters. On the other hand, laboratory synthesis of very fine magnetic particles is often plagued by the difficulty of producing narrow grain size distributions and, moreover, by clustering due to magnetostatic interactions that strongly affect the bulk magnetic properties. For example, Maher [1988] synthesized various batches of superparamagnetic magnetite grains with different size distributions. However, the susceptibility values were all $\chi<7$ (SI), much lower than theoretically expected.

An exceptionally suitable rock unit for the study of grainsize-dependent magnetic properties appears to be Yucca Mountain Tuff, characterized previously by Schlinger et al. [1988], and the subject of this investigation. As we will show, samples of this rock unit possess very narrow size distributions spanning the SP/SSD boundary, enabling us to test the predictions of Néel's theory concerning the frequency dependence of susceptibility.

\section{Theory}

The theoretical basis for the characterization of small magnetic particles was established by Néel [1949], who characterized the stability of single-domain (SD) grains in terms of a relaxation time $\tau$.

$$
\tau=\tau_{0} \exp (K V / k T)
$$

which depends on a magnetic anisotropy constant $K$, particle volume $V$, thermal energy $k T$, and a time constant $\tau_{0}$. Estimates for $\tau_{0}$ vary by orders of magnitude but it has recently been concluded [Worm, 1998] that for magnetite grains near the SP/SSD boundary, $\tau_{0}$ is most likely of the order of $10^{-9} \mathrm{~s}$. This is also the value that Moskowitz et al. [1997] determined for maghemite particles in magnetoferritin.

For uniaxial particles whose magnetization can only reverse by coherent rotation the anisotropy constant can be related to the microscopic coercivity $H_{k}$ and saturation magnetization $M_{s}$ :

$$
K=\mu_{0} H_{k} M_{S} / 2 .
$$

The anisotropy field $H_{k}$ can be calculated for ensembles of non-interacting SSD particles from the macroscopic coercivity $H_{c}$ via the theory of Stoner and Wohlfarth [1948]:

$$
H_{k}=2.09 H_{C} \text {. }
$$

The calculation of a blocking volume for a given time constant is straightforward, assuming that the coercivity is independent of volume:

$$
V_{b}=\frac{2 k T}{\mu_{0} H_{k} M_{S}} \ln \left(\tau / \tau_{0}\right) .
$$

The susceptibilities of superparamagnetic grains increase linearly (to a good approximation) with volume up to the SP/SSD threshold. We define the threshold volume in terms of the characteristic measurement time $\tau_{m}$ (for ac susceptibility measurements, $\tau_{m}=1 /\left(2 f_{m}\right)$, where $f_{m}$ is the measurement frequency). SP particles, smaller than the blocking volume for $\tau_{m}$, have susceptibilities given by [Néel, 1949]

$$
\chi_{s p}=\frac{\mu_{0} V M_{s}^{2}}{3 k T} .
$$

This is strictly true for the dc susceptibility only. For ac susceptibilities, (5) is modified by a frequency-dependent term [e.g., Worm, 1998]:

$$
\chi_{s p}=\frac{\mu_{0} V M_{s}{ }^{2}}{3 k T\left(1+\omega^{2} \tau^{2}\right)} .
$$

with $\omega=2 \pi f_{m}$.

Ensembles of noninteracting SSD particles, with volumes greater than $V_{b}\left(\tau_{m}\right)$ have susceptibilities [Stoner and Wohlfarth, 1948]

$$
\chi_{s d}=\frac{2 M_{s}}{3 H_{k}} .
$$


It is thus possible to model the temperature and frequency dependence of susceptibility provided the parameters saturation magnetization, coercivity, and volume (distributions) are known.

\section{Materials and Method}

\subsection{Sample Description}

The samples under study are tuffs from the Tiva Canyon member of the Paintbrush Tuff at Yucca Mountain (Nevada), and were kindly provided by C. M. Schlinger. Ashflow tuffs are volcaniclastic rocks, emplaced at high temperature, and formed as a consequence of explosive volcanism. Emplacement temperatures were $500-700^{\circ} \mathrm{C}$, and the base of the flow cooled to $20^{\circ} \mathrm{C}$ within a few weeks, but the cooling rate decreases with increasing stratigraphic height, and at $10 \mathrm{~m}$ above the base, temperatures remained high for tens of years [Schlinger et al., 1988]. The volcanic glass contains very fine-grained $\mathrm{Fe}$ oxides that formed by nucleation and growth, and the grain size increases systematically with distance from the base. The precipitates have been characterized by transmission electron microscopy and X-ray diffraction analysis as lath-shaped (uniaxial prolate) particles of cubic Fe oxides with $<10 \% \mathrm{Ti}$ content [Schlinger et al., 1988]. Eick and Schlinger [1990] measured the susceptibility and its frequency dependence at room temperature on eight tuff samples with different narrow grain size distributions, ranging from small superparamagnetic to stable singledomain grains. Three of these samples were selected for this study, and the grain sizes of the magnetic oxides are $5 \times 18$ $\mathrm{nm}$ (CS 916), $7.5 \times 37 \mathrm{~nm}$ (CS 915), and $8.5 \times 45 \mathrm{~nm}$ (CS 914) (C. M. Schlinger, personal communication, 1989) [Eick and Schlinger, 1990]. The sample names are adopted from their study.

\subsection{Measurements}

All magnetic measurements with different instruments have been performed on the same three specimens of $\approx 100$

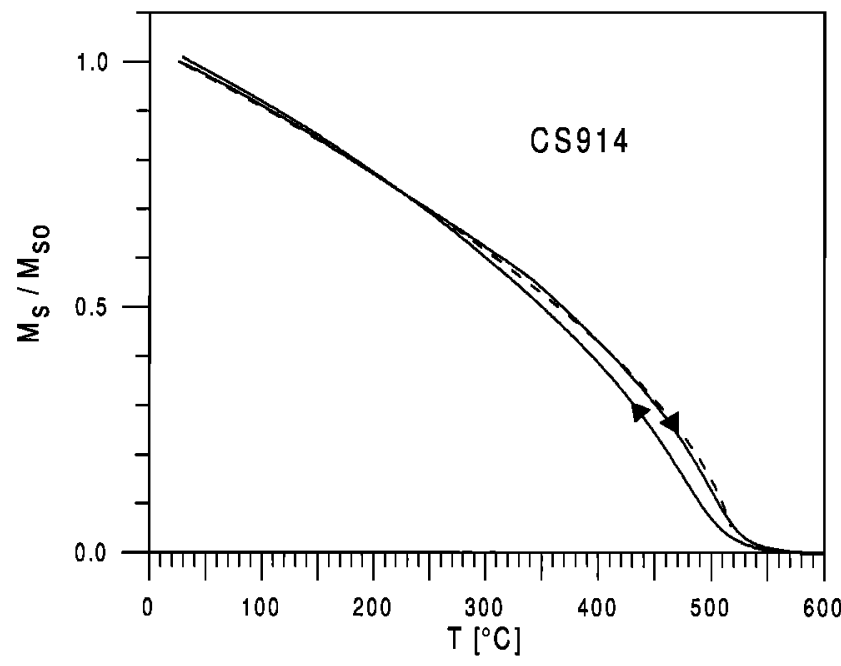

Figure 1. Thermomagnetic curve $\left(M_{5}-T\right)$ for CS 914 normalized to value $M_{\mathrm{so}}$ at $25^{\circ} \mathrm{C}$, with an applied field of 0.3 $\mathrm{T}$, heating and cooling at $20 \% \mathrm{~min}$ in $\mathrm{He}$ gas. The Curie temperature determined as average from heating and cooling legs is $T_{c}=521{ }^{\circ} \mathrm{C}$. The dashed line is an analytical approximation, $M_{s} / M_{\mathrm{so}} \propto\left[\left(T_{c}-T\right) /\left(T_{c}-T_{\mathrm{o}}\right)\right]^{06}, T_{0}=298 \mathrm{~K}$. mg weight, except for the experiments which required heating above room temperature for which separate pieces were used. A thermomagnetic curve was measured for Curie temperature determination with a Princeton Measurement Systems MicroMag vibrating-sample magnetometer ( $\mu$-VSM). Hysteresis loops were measured at room temperature with a VSM, and at 5 - $30 \mathrm{~K}$ with a Quantum Design SQUID magnetometer (MPMS). Acquisition and dc demagnetization of isothermal remanent magnetizations (IRM) were measured at $20 \mathrm{~K}$ with the MPMS as well. Thermal demagnetizations of a thermoremanence (acquired by cooling from $300 \mathrm{~K}$ to $5 \mathrm{~K}$ in a field of $5 \mathrm{mT}$ ) and of a saturation remanence were also measured with the MPMS magnetometer in the temperature range $5-320 \mathrm{~K}$. The frequency and temperature dependence of susceptibility were measured with a Lakeshore susceptometer (at frequencies ranging from $40 \mathrm{~Hz}$ to $4 \mathrm{kHz}$, temperatures in the range $20-300 \mathrm{~K}$, and ac fields of $240 \mathrm{~A} / \mathrm{m}$ ), and with the MPMS (at frequencies between 0.1 $\mathrm{Hz}$ and $1 \mathrm{kHz}$ in fields of $279 \mathrm{~A} / \mathrm{m}$ in the same temperature range). Susceptibilities above room temperature were measured with a Kappa-bridge (KLY-3, AGICO) operating at a frequency of $875 \mathrm{~Hz}$ and with a field of $300 \mathrm{~A} / \mathrm{m}$.

\section{Experimental Results}

\subsection{Thermomagnetic Curve}

A thermomagnetic curve was measured on the tuff sample CS 914 (Figure 1), in an applied field of $0.3 \mathrm{~T}$ while the sample was heated at $\sim 20^{\circ} / \mathrm{min}$. in a stream of He gas. The curve is almost reversible upon cooling. Part of the observed temperature hysteresis is instrument-related and results from the rather fast heating and cooling rates. The Curie temperature, determined as the average from the heating and cooling legs, is $T_{c}=521^{\circ} \mathrm{C}, \sim 50^{\circ} \mathrm{C}$ below that of pure magnetite.

\subsection{Hysteresis Loops}

At low temperatures $(<30 \mathrm{~K})$ all samples exhibit wide hysteresis and also significant paramagnetic magnetizations (Figure 2). At $5 \mathrm{~K}$ the paramagnetic signal is no longer linearly dependent on field because of saturation and ferrimagnetic ordering of some silicates. This complicates the extraction of the ferrimagnetic loop by subtraction of the (supposed linear) paramagnetic high-field slope, and therefore linear segments in the field range 0.5 to $1 \mathrm{~T}$ have been used for this purpose to yield the extracted loops of Figure 3. At room temperature all samples display essentially reversible magnetization versus field curves, indicating the expected superparamagnetic behavior (Figure 4a). Only CS 914, with the largest magnetic oxide grains of the three samples, exhibits a small hysteresis near the origin (Figure $4 b$ ).

The saturation magnetization values of the corrected lowtemperature loops (Figure 3) range from $M_{s}=0.36 \mathrm{~A} \mathrm{~m}^{2} / \mathrm{kg}$ (CS 914) to $M_{\mathrm{s}}=0.41 \mathrm{~A} \mathrm{~m}^{2} / \mathrm{kg}$ (CS 916), and assuming $M_{s} \approx$ $84 \mathrm{~A} \mathrm{~m}^{2} / \mathrm{kg}$ for the contained titanomagnetite minerals, a concentration of about $0.5 \mathrm{wt} \%$ can be calculated.

\subsection{IRM Acquisition and Backfield Remanence}

Comparison of isothermal remanent magnetization (IRM) acquisition and dc backfield remanence measurements can provide quantitative evidence for magnetostatic interactions [Wohlfarth, 1958]. The IRMs had to be measured in the SSD 

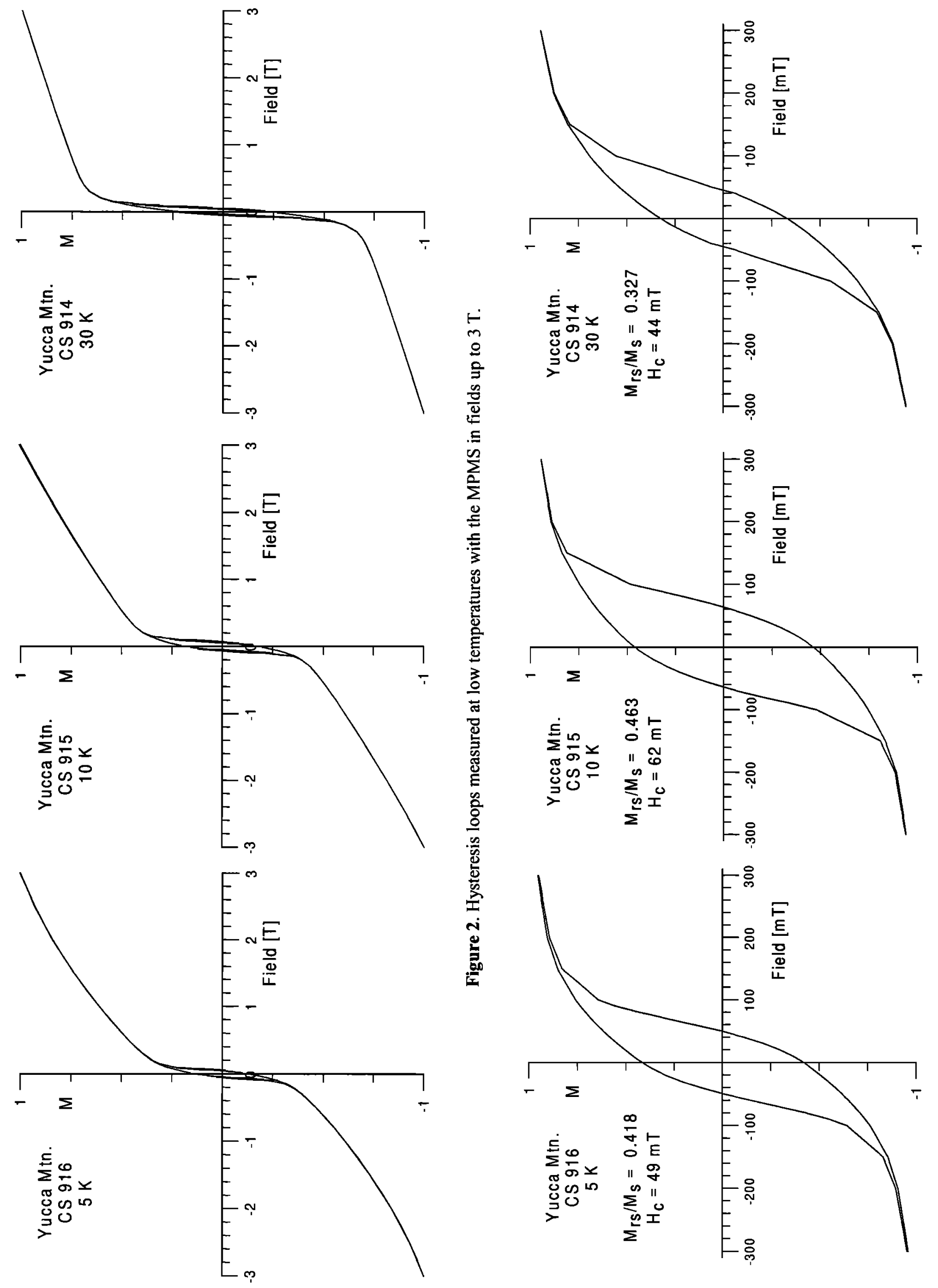

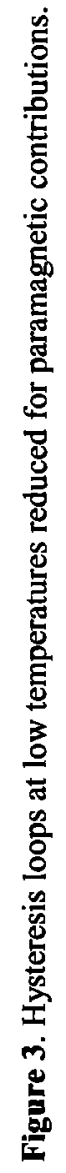



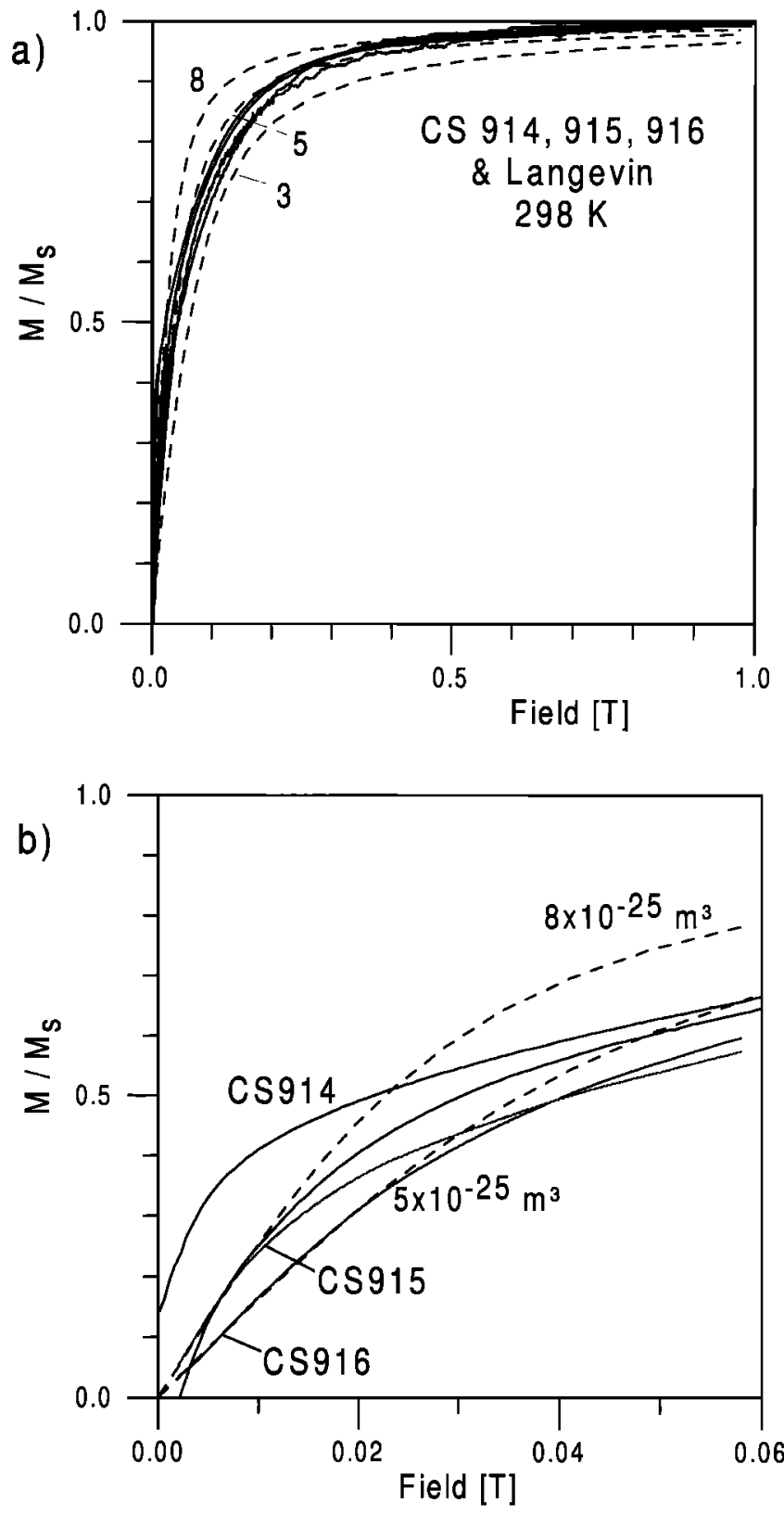

Figure 4. Magnetization curves for CS 914 , CS 915 and CS 916 at room temperature normalized to saturation magnetization $M_{s}$ together with Langevin curves calculated for volumes of 3,5 , and $8 \times 10^{-25} \mathrm{~m}^{3}$. (a) shows the full field range to $1 \mathrm{~T}$; (b) shows the low-field portion in detail.

state, and a temperature of $20 \mathrm{~K}$ was chosen for all samples (Figure 5). The reversed-field IRM data of Figure 5a are normalized by $1 / 2(1+$ IRM/SIRM). The same data are displayed in Figure 5b as a so-called Henkel plot [Henkel, 1964], where the IRM backfield values are plotted versus the IRM acquisition data with the field value as the common variable. Ideal noninteracting SD particles have a crossover point in Figure 5a at $R=0.5$, and the data in the Henkel plot fall on the indicated straight line. The experimental results are very close to the ideal noninteracting case, so that a theoretical evaluation of the susceptibility measurements does not have to be modified for interactions.

\subsection{Thermal Demagnetization}

The unblocking temperatures of the saturation remanence and the thermoremanence (TRM, 300 to $5 \mathrm{~K}$ ) are rather narrowly distributed and increase with increasing grain size from CS 916 to CS 914 (Figure 6). There is a noticeable highfield component with unblocking temperatures above $300 \mathrm{~K}$ that is absent for the TRM, but besides this the main unblocking occurs at the same temperatures for $M_{r s}$ and TRM, respectively. It is also evident from Figure 6 that the ratio $\mathrm{TRM} / M_{i s}$ decreases with increasing blocking temperature.

\subsection{Frequency and Temperature Dependence of Susceptibility}

The measured susceptibilities depend very strongly on frequency and temperature (Figure 7). There is excellent agreement between measurements performed on both instruments (for maintaining clarity duplicate measurements are not shown in Figure 7). The in-phase susceptibilities exhibit maxima that both decrease in magnitude and shift toward higher temperatures with increasing frequencies. The quadrature (out-of-phase) component $\chi$ " also exhibits maxima at temperatures that increase with frequency. The magnitudes of the $\chi$ " maxima do not decrease significantly with frequency, in contrast to the behavior of $\chi$. The quadrature component remains much smaller than the in-phase component for all frequencies and temperatures. The maxima of $\chi$ " occur always at lower temperatures than those of $\chi$ ' at corresponding frequencies.

The $\chi-T$ curve extending above room temperature shows no recognizable inflection at the Curie temperature (Figure 8). It is also less reversible than the $M_{s}$-T curve (Figure 1).

\section{Model}

The samples under study obviously possess narrow and distinct grain size distributions in the superparamagnetic grain size range. The magnetic mineral concentration in the rocks is low, $p \approx 0.5 \%$, and more importantly, the grains are not clustered (as in "dispersed" powders), with a minimum of magnetostatic grain interactions. The comprehensive set of measurements allows the complete characterization in terms of coercivity and volume distribution that is necessary for a theoretical evaluation of the susceptibility results.

\subsection{Saturation Magnetization}

The inference about the saturation magnetization is only indirect. From the previous mineralogical study it is clear that the magnetic minerals in the tuff are Ti-poor titanomagnetites [Schlinger et al., 1988]. From the thermomagnetic curve (Figure 1) a Curie temperature $T_{c}=521^{\circ} \mathrm{C}$ has been deduced. The curve is essentially reversible, indicating titanomagnetite rather than titanomaghemite. The small temperature hysteresis results mainly from the finite heating and cooling rates of $20 \%$ min. From several studies on synthetic titanomagnetites [see, e.g., Dunlop and Özdemir, 1997] it can be determined that a Curie temperature of $521^{\circ} \mathrm{C}$ corresponds to an ulvöspinel content in the titanomagnetite of $x \approx 0.1$, in agreement with the earlier characterization [Schlinger et al., 1988]. A saturation magnetization $\sim 15 \%$ lower than that of pure magnetite is thus assumed, $M_{\S}(0 \mathrm{~K})=429 \mathrm{kA} / \mathrm{m}$ and $M_{s}(298 \mathrm{~K})=407 \mathrm{kA} / \mathrm{m}$. 

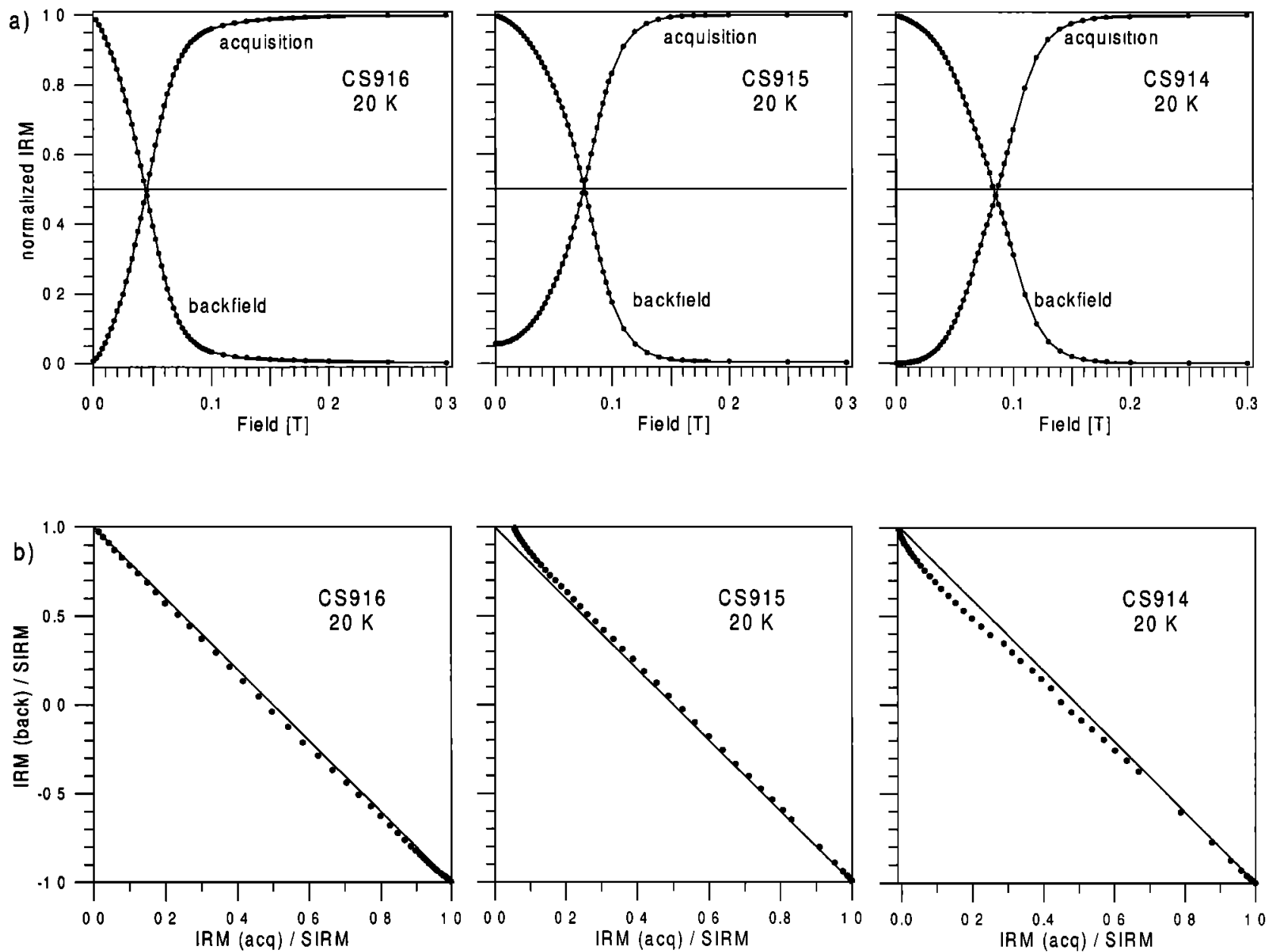

Figure 5. (a) Acquisition of isothermal remanent magnetization (IRM) normalized by saturation remanence and backfield IRM of the saturation remanence normalized by $1 / 2(1+$ IRM/SIRM). The crossover points of both curves occur near $R=0.5$. (b) Henkel plot displaying normalized IRM backfield versus IRM acquisition data. The straight line represents the theoretical Wohlfarth equation for noninteracting particles. All measurements at $20 \mathrm{~K}$.
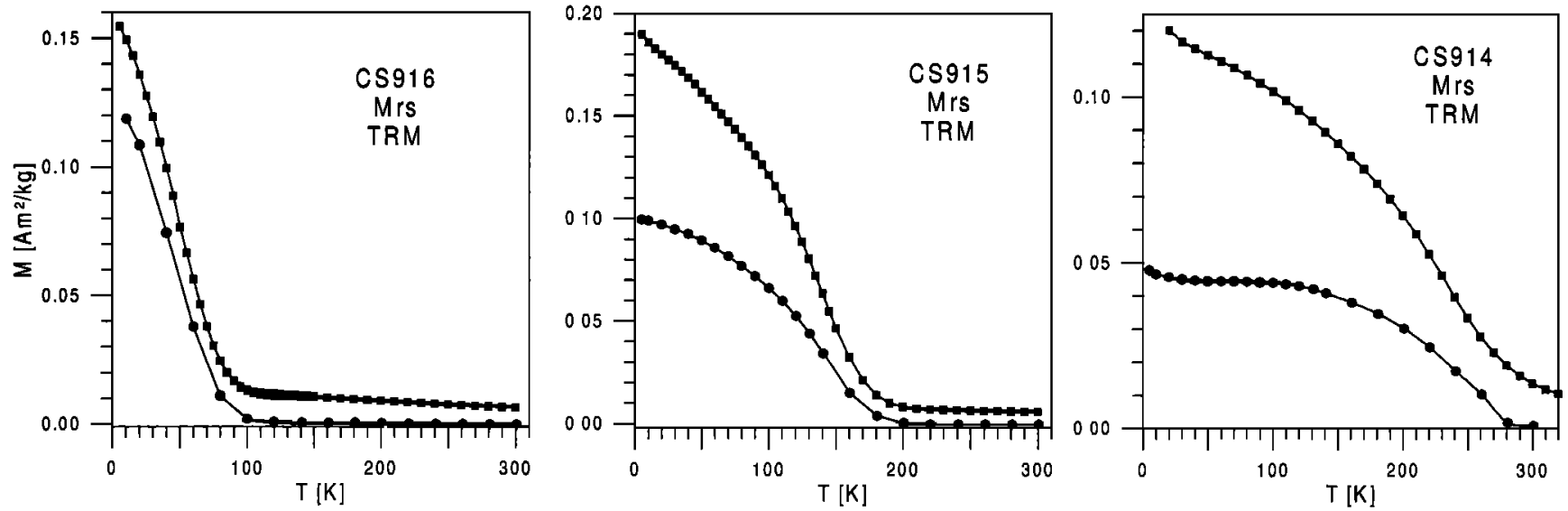

Figure 6. Thermal demagnetization of saturation magnetization $M_{r s}$ (squares) and thermoremanence TRM (circles) obtained by cooling from $300 \mathrm{~K}$ to $5 \mathrm{~K}$ in a $5 \mathrm{mT}$ field, measured with MPMS. 

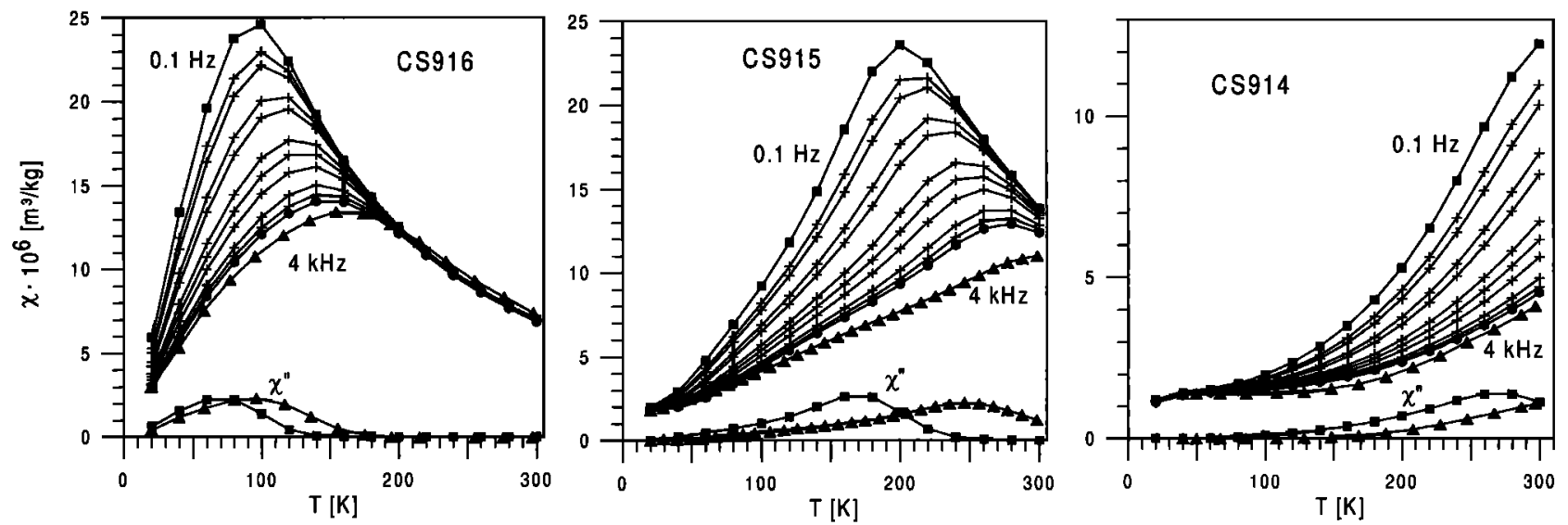

Figure 7. Experimental frequency and temperature dependence of susceptibility for CS $914, \mathrm{CS} 915$ and CS 916 in the temperature range 20 to $300 \mathrm{~K}$ measured with MPMS and Lakeshore. Shown is the in-phase component of the susceptibility for frequencies $0.1,0.5,1,5,10,50,100,200,500,750,1000$, and $4000 \mathrm{~Hz}$, and the quadrature component $\chi^{66}$ for the highest and lowest frequencies only.

\subsection{Calculation of Volume Distributions}

Under the assumption that the measured bulk coercivities at low temperature (Figure 3) represent the microscopic coercivities of all grains, the calculation of volume distributions is straightforward using equation (4) and the experimental unblocking curves. Fortunately, for this study the samples contain titanomagnetites rather than magnetite, for which the Verwey transition would result in a steep drop of remanences at $120 \mathrm{~K}$, thus preventing the successful determination of volume distributions. The saturation magnetization is assumed to vary as $M_{s}(T)=M_{s}(0)\left(1-C T^{3 / 2}\right)$ (Bloch's $T^{3 / 2}$ law) with $C=10^{-5}$. Preexponential time constants of $\tau_{0}=10^{-11}$ to $10^{-9} \mathrm{~s}$ have been employed in the calculations (equation (4)) in order to investigate the sensitivity of the results to the value of that parameter. The respective volume distributions derived from $M_{s s}$ and TRM unblocking are practically identical for CS 916 and for CS 915 (Figure 9). However, for CS 914 the distribution derived from unblocking of $M_{r s}$ contains a larger fine fraction than does the one derived from the TRM. The reason for this is not clear, but it can be suspected that magnetostatic interactions are more significant during demagnetization for the initial state of saturation remanence. The particle mean volumes $\left(\tau_{\mathrm{o}}=10^{-9} \mathrm{~s}\right)$ are $V_{\text {mean }}=0.8,1.8$, and $4 \times 10^{-24} \mathrm{~m}^{3}$ for CS 916 , CS 915, and CS 914, respectively, in excellent agreement with the values determined by transmission electron microscopy (TEM) [Schlinger et al., 1988]. For smaller $\tau_{0}$ values, slightly larger volumes are, of course, calculated.

The magnetization curve of superparamagnetic grains (as in Figure 4) may be expected to be described by the Langevin function $L(\alpha)=[\operatorname{coth}(\alpha)-1 / \alpha]$, where $\alpha=\mu_{0} V M_{\mathrm{s}} H / k T$ [e.g.

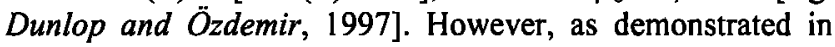
Figure $4 a$, the magnetization curves of the three samples are quite similar and fit between Langevin functions for volumes of 3 and $8 \times 10^{-25} \mathrm{~m}^{3}$. These have to be compared to the volumes derived from thermal unblocking $\left(8-40 \times 10^{-25} \mathrm{~m}^{3}\right)$. Langevin functions represent a good approximation to the measured curves only in low fields, up to $20 \mathrm{mT}$ for CS 916 and up to $8 \mathrm{mT}$ for CS 915, and not at all for CS 914 (due to hysteresis). For CS 916 the Langevin-derived mean volume (5 $\left.\times 10^{-25} \mathrm{~m}^{3}\right)$ is in fair agreement with the unblocking result $(8 \times$ $10^{-25} \mathrm{~m}^{3}$ ), but the difference is significant for CS 915. The reason for the poor overall fit is that the precondition for applying the Langevin function to superparamagnetic grains, namely, $K V / k T \ll 1$, is not met for these samples. For CS 916 , with the smallest grains, $K V / k T \approx 5$, and this ratio increases to 20 for CS 914 .

The volume distribution for CS 916 is a good approximation to a normal distribution. For CS 915 and CS 914 the distributions are skewed toward smaller grains. None of the calculated volume distributions are lognormal, although lognormal distributions are thought to be the norm for particles formed by nucleation and growth mechanisms [e.g., Heicklen, 1976; Walton, 1983].

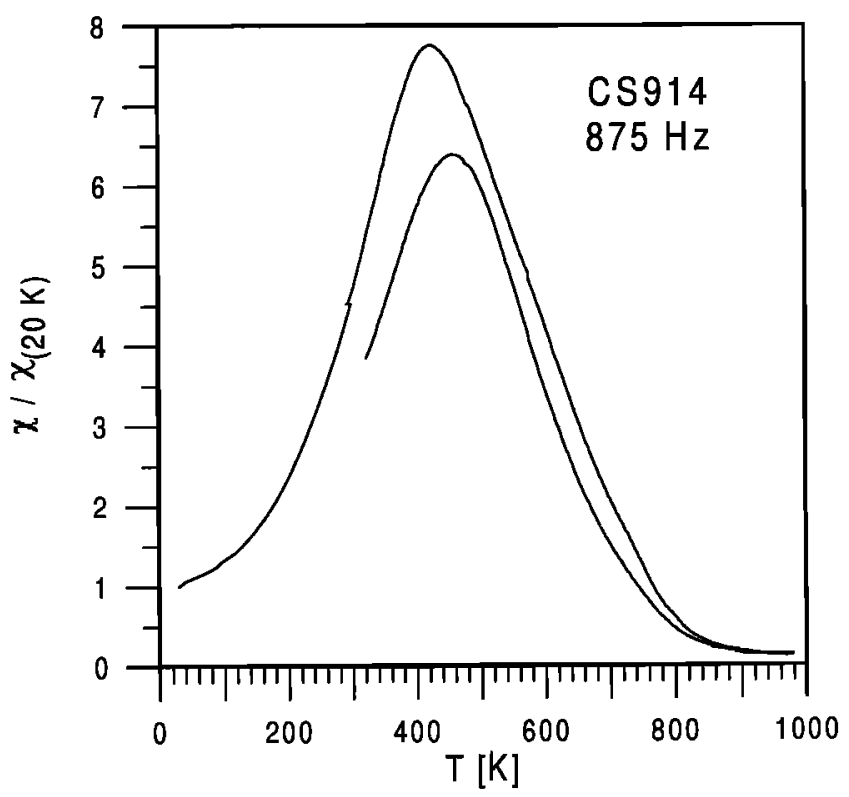

Figure 8. Susceptibility versus temperature for CS 914 measured with Kappabridge (above $300 \mathrm{~K}$ ) and Lakeshore (up to $300 \mathrm{~K}$ ). The lower curve is the cooling leg. 

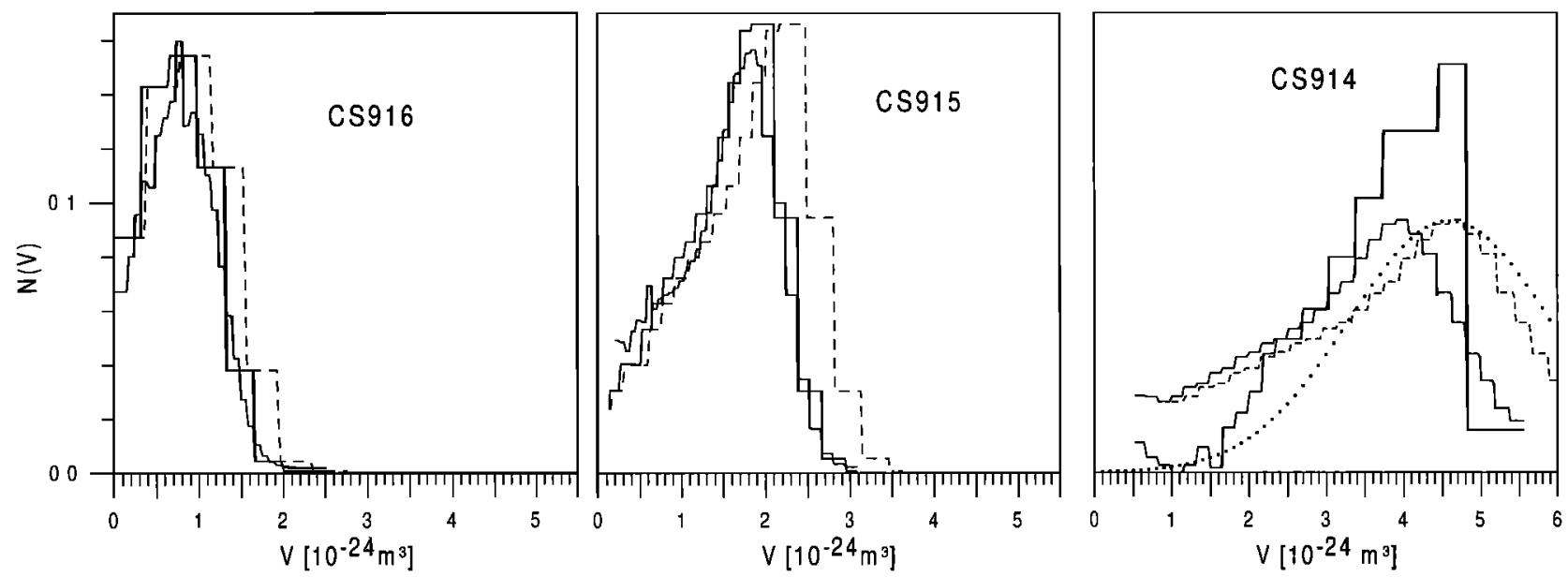

Figure 9. Calculated volume distributions for CS 914, CS 915 and CS 916 using thermal demagnetization results of Figure 6 and equation (4) with $\tau_{0}=10^{-9} \mathrm{~s}$. The narrow steps correspond to $M_{s \mathrm{~s}}$ and the wider subdivisions to TRM. The dashed lines represents results obtained for using $\tau_{0}=10^{-10} \mathrm{~s}$ for CS 916 and CS 915 , and $\tau_{0}=10^{-11} \mathrm{~s}$ for CS 914 . The dotted curve in CS 914 is a normal distribution approximation.

\subsection{Theoretical Frequency and Temperature Dependence of Susceptibility}

The susceptibilities of the three distinctively different superparamagnetic grain assemblages have been calculated numerically, using the experimentally determined saturation magnetization, coercivities, and volume distributions, for the highest and lowest experimental frequencies and as a function of temperature. The volume distributions derived from unblocking of the saturation remanence have been used, rather than those derived from the thermoremanence, because of the narrower measurement temperature intervals. As before, a variation of $M_{s}(T)=M_{s}(0)\left(1-C T^{3 / 2}\right)$ with $C=10^{-5}$ is assumed below room temperature, and for higher temperatures $M_{\mathrm{s}}$ and $H_{k}$ are assumed to vary proportionally with $\left[\left(T_{c^{-}}-T\right) /\left(T_{c^{-}} T_{0}\right)\right]^{06}, T_{o}=298 \mathrm{~K}$ (the best fit to the curve of Figure 1), as in the work by Worm et al. [1988], where an exponent 0.43 was found to be the best fit above $300^{\circ} \mathrm{C}$. In the calculations it was also assumed that for a given temperature, grains with relaxation times $\tau<1 /\left(2 f_{m}\right)$ have susceptibilities given by equation (5), while grains with $\tau>$ $1 /\left(2 f_{m}\right)$ have susceptibilities of stable single domain grains (equation (7)). This is not quite precise for relaxation times near $1 /\left(2 f_{m}\right)$, but including a relaxation-time-dependent parameter (equation (6)) in the susceptibility versus temperature calculations produces numerical problems. Nevertheless, it may be considered as an acceptable simplification because of the overall good agreement between experimental and calculated susceptibilities (Figure 10). Still there are systematic differences between measured and calculated curves: measured low-temperature susceptibilities are higher than those calculated for CS 916 and CS 915, and the computed maxima occur at higher temperatures than the measured maxima, the difference being larger for $4 \mathrm{kHz}$. This is true for both preexponential time constants, $\tau_{0}=10^{-9} \mathrm{~s}$ and $10^{-10} \mathrm{~s}$. Including the $\left(1+\omega^{2} \tau^{2}\right)$ term (equation (6)) would
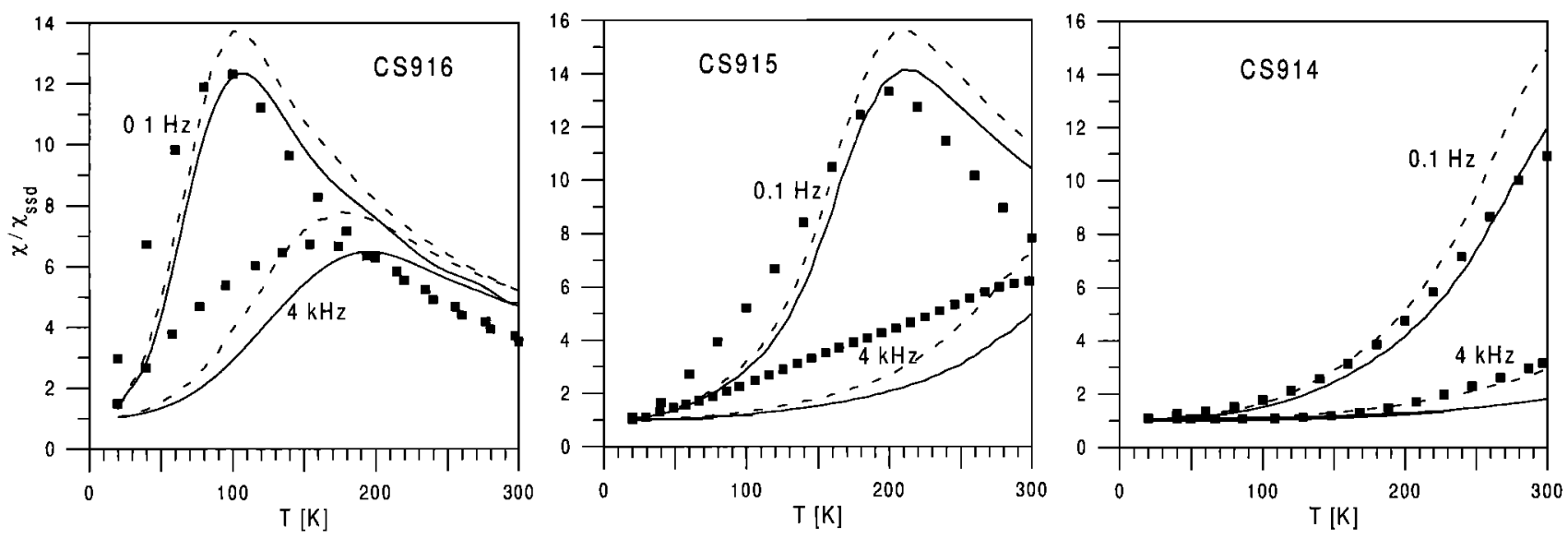

Figure 10. Calculated (lines) and experimental (squares) temperature dependence of susceptibility in the temperature range 20 to $300 \mathrm{~K}$ for frequencies $0.1 \mathrm{~Hz}$ and $4 \mathrm{kHz}$. Solid lines represent results obtained for calculations using $\tau_{\mathrm{o}}=10^{-9} \mathrm{~s}$, and dashed lines represent results using $\tau_{\mathrm{o}}=10^{-10} \mathrm{~s}$ for CS 915 and CS 916 or $\tau_{0}=10^{-11} \mathrm{~s}$ for $\operatorname{CS} 914$. 
lower the amplitudes but not alter the shape of the curves. For CS 914 at $4 \mathrm{kHz}$, an excellent fit is obtained with $\tau_{0}=10^{-11} \mathrm{~s}$ rather than with $\tau_{0}=10^{-9} \mathrm{~s}$, but for $0.1 \mathrm{~Hz}$ the calculated amplitude is larger than measured. Inclusion of the $\left(1+\omega^{2} \tau^{2}\right)$ factor, however, would result in lower values.

The calculated temperature dependence of susceptibility for $\tau_{0}=10^{-9} \mathrm{~s}$ and up to $750 \mathrm{~K}$ compares well with the experimental data for CS 914 (Figure 11) as far as the peak susceptibility and its temperature are concemed. The calculated curve, however, is broader than the measured one. When using $\tau_{0}=10^{-11} \mathrm{~s}$, the low-temperature susceptibilities are better approximated, but the peak height is much larger. Again, we expect that inclusion of the $\left(1+\omega^{2} \tau^{2}\right)$ term would improve the agreement between measured and modeled curves.

The modeled frequency dependence for CS 914 at room temperature, for $\tau_{\mathrm{o}}=10^{-9} \mathrm{~s}$, exhibits a stronger decrease with more curvature than the measured frequency dependence (Figure 12). For $\tau_{\mathrm{o}}=10^{-11} \mathrm{~s}$ a nearly perfect fit is obtained for frequencies above $50 \mathrm{~Hz}$; toward lower frequencies, the deviation increases. When using an analytical approximation for the volume distribution, it was possible to include the $\left(1+\omega^{2} \tau^{2}\right)$ term (equation (6) instead of equation (5)) in the calculations. A normal distribution, $N(V)=\exp \left[-\left(V-V_{m}\right)^{2} / 2 \sigma^{2}\right]$, $V_{m}=4.6 \times 10^{-24} \mathrm{~m}^{3}, \sigma=1.3 \times 10^{-24} \mathrm{~m}^{3}$, has been employed (Figure 9). Then the differences at low frequencies are decreased but at the expense of a poorer fit at high frequencies (Figure 12).

\section{Discussion and Conclusions}

The magnetic measurements of this study, together with the earlier microscopic characterization of Schlinger et al. [1988] and the previous susceptibility results obtained by Eick and Schlinger [1990] demonstrate that the Yucca Mountain Tuffs contain model superparamagnetic titanomagnetite

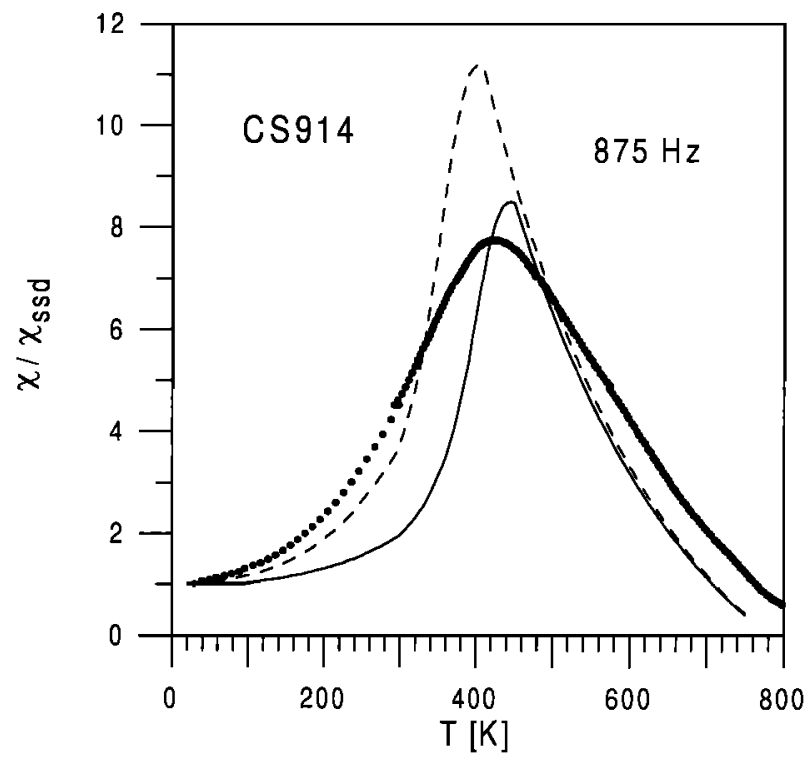

Figure 11. Calculated (lines) and experimental (circles) temperature dependence of susceptibility for CS 914 and $\mathrm{f}=875 \mathrm{~Hz}$ for temperatures up to $800 \mathrm{~K} . \tau_{\mathrm{o}}=10^{-9} \mathrm{~s}$ (solid line) and $\tau_{0}=10^{-11} \mathrm{~s}$ (dashed line).

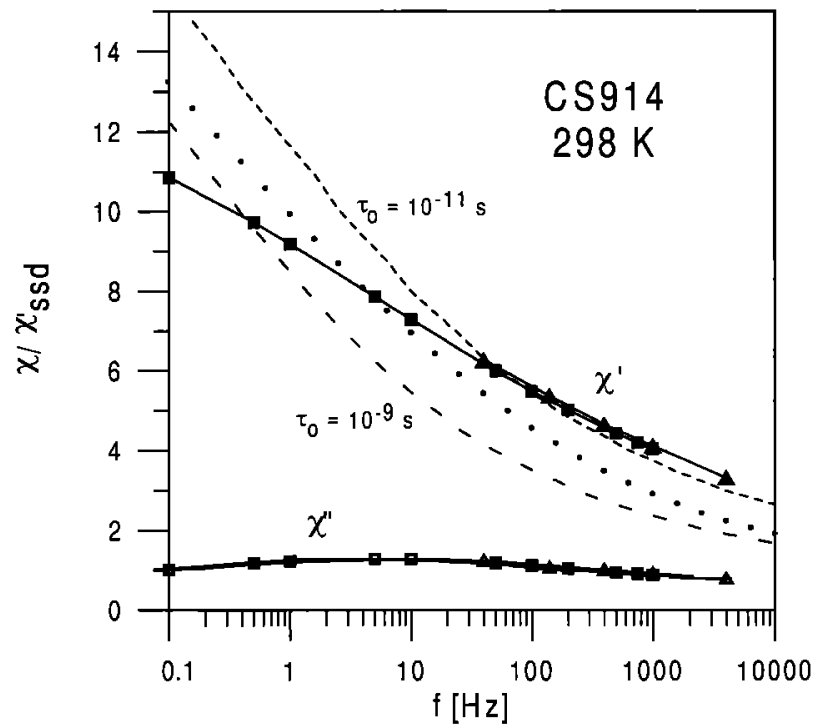

Figure 12. Frequency dependence of in-phase $\left(\chi^{\prime}\right)$ and quadrature $(\chi$ ") susceptibilities at room temperature for CS 914. Experimental results were obtained with MPMS (squares) and Lakeshore (triangles). Calculations of $\chi$ ' are with $\tau_{0}=10^{-9} \mathrm{~s}$ and $\tau_{0}=10^{-11} \mathrm{~s}$ for the volume distributions derived from unblocking of $M_{n}$ (eq. 5) and for a normal distribution approximation (dotted line) (equation (6), Figure 9).

grains of distinctly different sizes. The grain-size-controlling parameter is stratigraphic position within the ashflow sheet above its rapidly cooled base and hence the temperature at which the titanomagnetites were nucleated and grown from volcanic glass [Schlinger et al., 1988].

The shape of the hysteresis loops measured at low temperatures resembles the theoretical curves for ensembles of single-domain grains calculated by Stoner and Wohlfarth [1948]. Our assumption of identical microcoercivities for all grains thus appears well justified.

Thermal demagnetization of a saturation remanence or thermoremanence (Figure 6) is obviously an excellent method for obtaining volume distributions of single-domain grain samples. Agreement between distributions derived from $M_{n}$, and TRM demagnetization for the two samples with the smaller grains is excellent; at present, it is not clear why sample CS 914, with main blocking temperatures just below room temperature, yields somewhat discordant results. The larger $M_{r s}$ TRM ratio for CS 914 (Figure 6) could suggest that magnetostatic interactions may be more pronounced during $M_{\text {s s }}$ demagnetization; however the Wohlfarth test for magnetostatic interactions by IRM measurements (Figure 5) indicates almost noninteracting grains. It is also evident from the magnetization curve at room temperature (RT) that a grain fraction of CS 914 extends into the stable single-domain regime resulting in wasp-waisted hysteresis. The TRM acquired during cooling from 300 to $5 \mathrm{~K}$ and the thermal unblocking measurements have thus not affected the largest grains.

The comparison of the RT magnetization curves with Langevin curves based on the volume distributions derived from thermal demagnetization demonstrates that in the case 
where $K V / k T \gg 1$, it is not possible to obtain a volume estimate by a Langevin fit to the measured magnetization curve. It is only for the sample containing the smallest grains, for which $K V / k T \approx 5$, and only in the low field regime, that the Langevin volume is comparable to the one derived from unblocking. Surprisingly, the overall shapes of the magnetization curves of the three samples under study are quite similar at room temperature (Figure 4a) despite their distinctly different magnetic grain volumes. Tauxe et al. [1996] found that inclusion of anisotropy energy in their numerical models resulted in magnetization curves approaching saturation more slowly than the Langevin function, for particle diameters greater than about $8 \mathrm{~nm}$, and varying only slightly with particle size between 10 and $25 \mathrm{~nm}$. Nonetheless, while in their calculations the magnetization curve for $10 \mathrm{~nm}\left(10^{-24} \mathrm{~m}^{3}\right)$ anisotropic particles roughly coincides with the corresponding Langevin curve, we find strong deviations by factors up to 10 in volume toward seemingly smaller grain sizes for these samples (Figure 4a).

The experimental results show that the frequency dependence of susceptibility has a maximum at a slightly higher temperature than the dc blocking temperature and that the maximum shifts toward higher temperatures with increasing frequency while the amplitude decreases. This is quite well modeled on the basis of Neel's theory, using the experimentally determined volume distributions. However, discrepancies between modeled and measured susceptibilities exist, and the physical causes are not obvious. It could be suspected that CS 916 contains a population of extremely small grains with unblocking temperatures $<5 \mathrm{~K}$ that are thus not detected during thermal demagnetization. However, this fraction cannot be large because $M_{r s} / M_{s}=0.42$ at $5 \mathrm{~K}$ (Figure 3 ), close to the ideal SSD value. Alternatively, the assumption of a size-independent anisotropy may be invalid. If the grains' shape anisometry varies with size, the calculated volume distributions would be skewed. Also, the measured coercivities are incompatible (smaller) with the model of coherent magnetization reversal and the observed grain elongations [Schlinger et al., 1988]. Nonuniform modes, such as fanning [e.g., Dunlop and Özdemir, 1997], must prevail during hysteresis and possibly also in the weak field regime.

The susceptibility of CS 914 is best modeled with $\tau_{0}=$ $10^{-11} \mathrm{~s}$ instead of $10^{-9} \mathrm{~s}$, in contrast to CS 915 and CS 916 for which a decrease below $\tau_{\mathrm{o}}=10^{-9} \mathrm{~s}$ does not improve the model calculations (Figure 9). Measured susceptibilities above $500 \mathrm{~K}$ are larger than calculated (Figure 11), presumably caused by the grain size fraction with blocking temperatures $>320 \mathrm{~K}$ (Figure 6) and thus not considered in the model. Similarly, the calculated peak intensity would be reduced by a broadened distribution and also by the frequency factor $\left(1+\omega^{2} \tau^{2}\right)$. All calculations of susceptibility versus frequency at room temperature result in concave-up curves of $\chi[\log (f)]$, rather than the observed approximately straight decrease (Figure 12), possibly a result of an unaccounted distribution of coercivities which would smooth the calculated curves [Worm, 1998].

Questions remain about the nature of the preexponential time constant $\tau_{0}$, concerning its value but also its assumed independence of volume and temperature. It was assumed that $\tau_{\mathrm{o}}=10^{-9} \mathrm{~s}$, but for CS $914, \tau_{\mathrm{o}}=10^{-11} \mathrm{~s}$ results in better agreement with the experiments. On the other hand, for the samples containing smaller grains, $\tau_{0}<10^{-9} \mathrm{~s}$ does not improve the fit. It may be suspected that $\tau_{0}$ is not constant but is size- and temperature-dependent, as predicted by Brown [1959]. However, no attempt has been undertaken in the calculations of this study to treat $\tau_{0}$ as a variable.

It is evident that superparamagnetic grains do not necessarily exhibit a frequency dependence of susceptibility at all temperatures. The conclusion is sometimes drawn in rock magnetic studies that zero frequency dependence indicates the absence of SP grains; this should be regarded with caution. On the other hand, a frequency dependence $\chi_{\mathrm{fd}} \approx 15 \%$ is not necessarily indicative of a pure SP assemblage of grains. Although the results of several studies may suggest so [Thompson and Oldfield, 1986; Dabas et al., 1992; Forster et al., 1994; Dearing et al., 1996], 15\% is not an absolute upper limit for $\chi_{\mathrm{fd}}$. Sample CS 914 possesses a frequency dependence of $\chi_{\mathrm{fd}} \approx 30 \%$, for $f_{\mathrm{lf}}=400 \mathrm{~Hz}$ and $f_{\mathrm{hf}}=4 \mathrm{kHz}$. This value may indeed constitute one of the highest ever reported for the frequency dependence of susceptibility due to Néel relaxation. The much studied "model" superparamagnet ferritin has a maximum $\chi_{\mathrm{fd}} \approx 25 \%$ at $10 \mathrm{~K}$ (and $\chi_{\mathrm{fd}} \approx 0 \%$ at room temperature) [Kilcoyne and Cywinski, 1995]. Measurements on nanometer-sized $\mathrm{Fe}$ particles exhibit frequency dependences of $\chi_{\mathrm{fd}}<20 \%$ [Dormann, 1981; Bødker et al., 1998]. For ferrofluids, larger frequency dependences of susceptibility with $\chi_{\mathrm{fd}}>40 \%$ have been reported [Konn et al., 1995]; however, these are mainly due to Brownian relaxation by particle rotation in the liquid carrier.

In the Yucca Mountain samples the volume distributions are very narrow, with relatively sharp upper limits (Figure 9), and these characteristics of the size distribution are primariy responsible for the unusually strong frequency dependence. At sufficiently low frequency the blocking volume is near the upper end of the volume distribution, and most or all of the particles are in the SP state. $V_{b}$ decreases with increasing frequency according to equation (4), and as it sweeps through the volume distribution of the sample, susceptibility drops as particles undergo the SP-SSD transition. The initial rate of change in susceptibility with increasing frequency is therefore directly related to the sharpness of the upper end of the size distribution. The reason for the apparent ceiling on $\chi_{\mathrm{fd}}$ in many natural materials is most likely due to the preponderance of lognormal size distributions in soils and other materials exhibiting the apparent $15 \%$ limit. Lognormal distributions are characterized by a gradually diminishing tail at the upper end of the distribution, resulting in a broad range of blocking frequencies and thus in limited frequency dependence.

In the calculations of Eyre [1997], excessive frequency dependence resulted from various factors including a large SP-SSD threshold, a discontinuous decrease in susceptibility across the threshold, and omission of the effects of coercivity distributions. Nevertheless, a major conclusion from that paper is still valid and important: frequency dependence of susceptibility, when based on measurements at only two frequencies and one temperature, provides a quite limited and highly nonunique view of the size distribution of ferromagnetic grains in a sample. In contrast, measurements over a range of frequencies and temperatures allow fairly precise quantification of the distribution of particle sizes in the SP interval.

The Yucca Mountain samples are extraordinary examples of noninteracting superparamagnetic and single-domain assemblages. They exhibit ideal Néel behavior rarely observed in either synthetic samples (which nearly always 
show effects of interaction due to imperfect dispersal) or natural materials (which tend to have rather wide distributions of particle sizes). The rarity of such ideal behavior is not due to any undiscovered flaw in the theory but rather to the rarity of ideal assemblages.

Acknowledgments. We are grateful to Charly Schlinger who provided the Yucca Mountain Tuff samples. Reviews by M. Dekkers, F. Heller and C. Laj significantly improved the quality of the manuscript. Financial support from the "Deutsche Forschungsgemeinschaft" is gratefully acknowledged. This is IRM contribution 9902 . The IRM is supported by grants from the National Science Foundation and the W. M. Keck Foundation.

\section{References}

Bødker, F., S. Mørup, M.S. Pedersen, P. Svedlindh, G.T. Jonnsson, J.L. Garcia-Palacios, F.J. Lazaro, Superparamagnetic relaxation in $\alpha$-Fe particles, J. Magn. Magn. Mater., 177-181, 925-927, 1998.

Brown, W.F., Relaxation behavior of fine magnetic particles, $J$. Appl. Phys., 30, 130-132, 1959.

Dabas, M., A. Jolivet, and A. Tabbagh, Magnetic susceptibility and viscosity of soils in a weak time varying field, Geophys $J$. Int., 108, 101-109, 1992.

Dearing, J.A., R.J.L. Dann, K. Hay, J.A. Lees, P.J. Loveland, B.A. Maher, and K. O'Grady, Frequency-dependent susceptibility measurements of environmental materials, Geophys. J. Int., 124 , 228-240, 1996.

Dormann, J.L., Le phénomène de superparamagnétisme, Rév. Phys. Appl., 16, 275-301, 1981.

Dunlop, D.J., and Ö. Özdemir, Rock Magnetism: Fundamentals and Frontiers, 573 pp., Cambridge Univ. Press, New York, 1997.

Eick, P.M., and C.M. Schlinger, The use of magnetic susceptibility and its frequency dependence for delineation of a magnetic stratigraphy in ash-flow tuffs, Geophys. Res. Lett., 17, 783-786, 1990.

Eyre, J.K., Frequency dependence of magnetic susceptibility for populations of single-domain grains, Geophys. J. Int., I29, $209-$ $211,1997$.

Forster, T., M.E. Evans, and F. Heller, The frequency dependence of low field susceptibility in loess sediments, Geophys. J. Int., 118, 636-642, 1994.

Heicklen, J., Colloid Formation and Growth, 327 pp., Academic, San Diego, Calif., 1976.

Henkel, O., Remanenzverhalten und Wechselwirkungen in hartmagnetischen Teilchenkollektiven, Phys. Status Solidi, 7, 919-924, 1964.
Kilcoyne, S.H. and R Cywinski, Ferritin: a model superparamagnet, J. Magn. Magn. Mater., 140-144, 1466-1467, 1995.

Konn, A.M., P.Laurent, P. Talbot, M. Le Floc'h, Study of relaxation and spin resonance in magnetic liquids, J. Magn. Magn. Mater., 140-144, 367-368, 1995.

Maher, B.A., Magnetic properties of some synthetic sub-micron magnetites, Geophys. J., 94, 83-96, 1988.

Moskowitz, B.M., R.B. Frankel, S.A. Walton, D.P.E. Dickson, K.K.W. Wong, T. Douglas, and S. Mann, Determination of the pre-exponential frequency factor for superparamagnetic maghemite particles in magnetoferritin, J. Geophys. Res., 102, 22671-22680, 1997.

Néel, L., Théorie du trainage magnétique des ferromagnétiques en grains fin avec application aux terres cuites, Ann. Géophys., 5, 99-136, 1949.

Schlinger, C.M., J.G. Rosenbaum, and D.R. Veblen, Fe-oxide microcrystals in welded tuff from southern Nevada: Origin of remanence carriers by precipitation in volcanic glass, Geology, $16,556-559,1988$.

Stephenson, A., Single domain grain distributions I. A method for the determination of single domain grain distributions, Physics of the Earth and Planetary Interiors, 4, 353-360, 1971.

Stoner, E.C., and E.P. Wohlfarth, A mechanism of magnetic hysteresis in heterogeneous alloys, Philos. Trans. R. Soc., Ser A, 240, 599-642, 1948.

Tauxe, L., T.A.T. Mullender, and T. Pick, Potbellies, wasp-waists, and superparamagnetism in magnetic hysteresis, J. Geophys. Res, $101,571-583,1996$.

Thompson, R., and F. Oldfield, Environmental Magnetism, 227 pp., Allen and Unwin, London, 1986.

Walton, D., Viscous magnetization, Nature, 305, 616-619, 1983.

Wohlfarth, E.P., Relations between different modes of acquisition of the remanent magnetization of ferromagnetic particles, J. Appl. Phys., 29, 595-596, 1958.

Worm, H.-U., On the superparamagnetic-stable single domain transition for magnetite, and frequency dependence of susceptibility, Geophys. J. Int., 133, 201-206, 1998.

Worm, H.-U., M. Jackson, P. Kelso, and S.K. Banerjee, Thermal demagnetization of partial thermoremanent magnetization, $J$. Geophys. Res., 93, 12,196-12,204, 1988.

M. Jackson, Institute for Rock Magnetism, Department of Geology and Geophysics, University of Minnesota, Minneapolis, MN 55455. (irm@geolab.geo.umn.edu).

H.-U. Worm, Federal Institute for Geosciences and Natural Resources, Hannover, Germany. (huworm@t-online.de)

(Received January 20, 1999; revised July 23, 1999; accepted August 10. 1999.) 